\section{HER-2-Negative Breast Cancer Limitations and Next-Generation Sequencing Technology Promises}

\section{TO THE EDITORS:}

On the basis of human epidermal growth factor receptor 2 (HER-2) status, patients with breast cancer are classified as having HER-2-negative or HER-2-positive disease. Prognosis of HER-2-positive, as compared to HER-2negative, disease is poorer. But by adding HER-2 signaling pathway inhibitors, including trastuzumab, to standard chemotherapy, we can change this poor outcome.

Given that more than three of four patients diagnosed with breast cancer have a HER-2-negative disease and yet in the absence of any deregulated signaling pathway having been discovered no targeted therapy can be applied, there is major interest in how best treat these patients. Several phase II and III randomized trials are underway to test many combinations of targeted agents. But the expectations are limited by the lack of the identification regarding which signaling pathways are activated in these HER-2-negative patients.

Therefore, translational research on HER-2-negative disease is currently attracting major interest. Mizell and colleagues evaluated whether chemokine receptor CXCR4 expression could be used as a prognostic factor. ${ }^{1}$ In their report in a recent issue of the Annals of Surgical Oncology, the authors analyzed CXCR4 levels from primary tumors of 115 HER-2-negative breast cancer patients with stage I to III disease. The authors report that overall survival and disease-free survival were far lower among 13 patients with CXCR4 overexpression than among 102 patients with low CXCR4 expression in primary tumors.

The authors propose the performance of either a prospective clinical trial and/or an external validation study to validate the prognostic significance of.CXCR4 expression. But the study is limited by the very small number of patients ( $n=13$ ) with CXCR4 overexpression. Comparative effectiveness research requires much larger samples than 12 cases, particularly when Kaplan-Meier survival curves and Cox proportional hazard ratio models are used for comparisons. Such comparisons need comparability of the two groups at baseline, including tumor stage, clinicopathologic factors, and treatment.

Currently, treatment of patients with HER-2-negative tumor is suboptimal. Given the high complexity and erogeneity of the breast, conventional single-protein-coding gene research has many limitations. There is a need to identify which other signaling pathways, beyond those in the epidermal growth factor receptor family (HER-1, HER2, HER-3, HER-4)—for example, Wnt/Notch-are activated and predict how their interactions in HER-2-negative disease affect outcome. This intracellular signaling pathways network understanding appears may now become feasible with the advent of next-generation DNA sequencing technology. These technological advances, including genomewide RNA, serial analysis of gene expression (SAGE), micro-RNAs, protein-DNA interactions, and comprehensive analyses of transcriptomes and interactomes, open new roads for the development of new markers and drugs against breast cancer and other solid tumors. $^{2-5}$

Georgios Baltogiannis, MD ${ }^{1}$, Christos Katsios, MD $^{1}$, George C. Zografos, $\mathrm{MD}^{2}$, and Dimitrios H. Roukos, $\mathrm{MD}^{1}$ ${ }^{1}$ Department of Surgery, Ioannina University School of Medicine, Ioannina, Greece;

${ }^{2} 1$ st Department of Surgery, University of Athens, Athens, Greece

e-mail: droukos@cc.uoi.gr

Published Online: 3 March 2010

(c) Society of Surgical Oncology 2010

\section{REFERENCES}

1. Mizell J, Smith M, Li BD, Ampil F, Chu QD. Overexpression of CXCR4 in primary tumor of patients with HER-2 negative breast cancer was predictive of a poor disease-free survival: a validation study. Ann Surg Oncol. 2009;16(10):2711-6.

2. Schadt EE. Molecular networks as sensors and drivers of common human diseases. Nature. 2009;461(7261):218-23.

3. Rockman MV. Reverse engineering the genotype-phenotype map with natural genetic variation. Nature. 2008;456:738-44.

4. Hahn WC, Weinberg RA. Modelling the molecular circuitry of cancer. Nat Rev Cancer. 2002;2(5):331-41.

5. Wood LD, Parsons DW, Jones S. The genomic landscapes of human breast and colorectal cancers. Science. 2007;318(5853): 1108-13. 